malaria vaccine and drug development. Sanaria Inc. USA, has devised methods of producing, characterising and shipping aseptic, purified, cryopreserved Plasmodium falciparum sporozoites (PfSPZ). Unlike in the past, the sporozoites can be shipped to any field site including malaria-endemic countries in sub-Saharan Africa. CHMI studies need cutting-edge laboratory capacity to allow screening of potential study participants for their eligibility, perform safety tests, efficacy testing of the investigation product, and determine immune markers, responses and parasite kinetics.

Methods The sites were identified within sub-Saharan Africa, based on local population, malaria epidemiology status and clinical research capacity. The laboratory assays, equipment and consumables were identified. The methods and procedures were optimised, standardised and documented. The laboratory technical team were selected and trained. EDCTP provided financial support whilst Sanaria Inc. provided technical support.

Results The capacity strengthening successfully supported CHMI studies in five sites in Africa namely; IHI, Bagamoyo, Tanzania; KEMRI CRC, Nairobi, Kenya; EGMVI, Malabo Equatorial Guinea; CERMEL, Lambarane, Gabon; KEMRI-CDC, Siaya-Kenya and KEMRI-WT, Kilifi Kenya. Capacity strengthening was aimed at clinical and immunology laboratory systems required for CHMI studies.

Conclusions As more CHMI studies are anticipated, vigorous capacity strengthening will be required for laboratory facilities in Africa to accelerate the evaluation of malaria vaccines, antimalaria drugs, diagnostic assays and assessment of host immune response to malaria infection.

\section{PA-008 THE QUEST FOR BUILDING LABORATORY CAPACITY TO SUPPORT CONTROLLED HUMAN MALARIA INFECTION (CHMI) STUDIES IN SUB-SAHARAN AFRICA: EXPERIENCE WITH FIVE SITES}

Kennedy Awuondo, ${ }^{1}$ Daniel Njenga, ${ }^{2}$ Elizabeth Nyakarungu, ${ }^{3}$ Pauline Titus, ${ }^{2}$ Awalludin Sutamihardja, ${ }^{4}$ Brett Lowe, ${ }^{1}$ Bernhards Ogutu, ${ }^{2}$ Peter Billingsley ${ }^{5}$. ${ }^{1}$ KEMRI-Wellcome Trust, Kenya; ${ }^{2}$ KEMRI (Coast), Kenya; ${ }^{3} / \mathrm{HI}$, Tanzania; ${ }^{4}$ Walter Reed, Indonesia; ${ }^{5}$ Sanaria Inc., United States of America

10.1136/bmjgh-2016-000260.47

Background Conducting Controlled Human Malaria Infection (CHMI) studies in sub-Saharan Africa presents unique challenges yet it provides enormous opportunities for fast-tracking 